\section{Tratamiento para lesiones de furcación Clase l: plastia furcal}

Alania Mallqui JA. Ceccarelli Calle JF. Delgado-Bravo M. Tratamiento para lesiones de furcación Clase I: plastia furcal. Rev Estomatol Herediana. 2011; 21(1):30-33.

\section{RESUMEN}

Los defectos en furcaciones representan un gran reto para los terapistas debido a sus características anatómicas y a su variabilidad en cuanto a la forma de respuesta al tratamiento. La pérdida de inserción se desarrolla en sentido horizontal o vertical del área. Un amplio rango de modalidades de tratamiento han sido dirigidos a mantener esta área anatómica: debridamiento mecánico no-quirúrgico y quirúrgico, plastia furcal, procedimientos de tunelización, hemisecciones, amputaciones radiculares y tratamiento regenerativos. El propósito del presente reporte de caso es mostrar la eficacia de la plastia furcal como tratamiento para lesiones de furcación Clase

Palabras clave: DEFECTOS DE FURCACIÓN / PÉRDIDA DE LA INSERCIÓN PERIODONTAL / PÉRDIDA DE HUESO ALVEOLAR.

Treatment of Class I furcation lesion: furcation plasty

ABSTRACT

Furcation defects represent a challenge for therapists due to their anatomical characteristics and its variability in the form of response to treatment. Consequently, loss attachment develops in horizontal and vertical direction in this area. A wide range of treatment modalities have been directed to maintain this anatomical area: non-surgical and surgical mechanical debridement, furcation plasty, tunneling procedures, hemisection, root amputation and regenerative therapy.

The purpose of this report is to show the effectiveness of the furcation plasty as a treatment for Class I furcation lesions.

Key words: FURCATION DEFECTS / PERIODONTAL ATTACHMENT LOSS / ALVEOLAR BONE LOSS.

\section{Jorge Alberto Alania Mallqui ${ }^{1}$ Juan Francisco Ceccarelli Calle ${ }^{2}$ Miguel Delgado Bravo²}

${ }^{1}$ Residente del Programa de Especialización en Peridoncia e Implantes Dentales.

2Docente del Departamento Académico de Clínica Estomatológica.

Fstomatológica.

Cayetano Heredia.

\section{Correspondencia}

Jorge Alberto Alania Mallqui Alfonso Ugarte 366, Lima 31 - Perú. Teléfono: 963533358

e-mail: jorgealberto87@ hotmail.com

Recibido : 17 de noviembre de 2010

Aceptado : 15 de enero de 2011

\section{Introducción}

El principal factor etiológico de la enfermedad periodontal es la presencia y maduración del biofilm bacteriano. El tratamiento de la enfermedad consiste principalmente en el debridamiento mecánico subgingival que conlleva al establecimiento de un medio ambiente bacteriano compatible con salud gingival (1).

Los estudios han demostrado que la periodontitis crónica puede ser tratada con gran éxito mediante la terapia quirúrgica y no quirúrgica. El mantenimiento a largo plazo de resultados favorables se logra mediante un adecuado control de placa durante la fase del tratamiento periodontal de soporte (2).

Los dientes multi-radiculares presentan características anatómicas únicas, las cuales representan un reto para el paciente y para el profesional debido a su inaccesibilidad para un buen control de placa $(3,4)$. En pacientes susceptibles a la enfermedad periodontal, la pérdida de inserción puede progresar hasta involucrar el área de furcación tanto en sentido vertical como horizontal. Hamp, Nyman y Lindhe (5) establecieron una clasificación de los defectos de furcación en sentido horizontal. El defecto clase I es aquel que al ser evaluado con una sonda periodontal de Nabers, ésta logra ingresar hasta $3 \mathrm{~mm}$ a través de la furcación; el defecto clase II corresponde a aquel en el cual la sonda logra ingresar más de $3 \mathrm{~mm}$ a través de la furcación sin atravesarla por completo y el defecto clase III es aquel en el cual la sonda periodontal atraviesa totalmente la zona de furcación.

Los estudios clínicos han demostrado que el compromiso del área de furcación en las molares conlleva a una menor respuesta al tratamiento no quirúrgico, además de una mayor predisposición de pérdida de in- serción (6-8).

El menor pronóstico de los dientes con compromiso de furcación es confirmado con el análisis de los resultados de estudios longitudinales. Hirschfeld y Wasserman en 1978 (9) demostraron tras un periodo de observación de 22 años, en pacientes que se encontraban en tratamiento periodontal de soporte, que un $7,1 \%$ de los dientes se perdieron por razones periodontales. De estos el 31\% eran dientes con compromiso de furcación (9-11).

Un amplio rango de modalidades de tratamiento se han propuesto para el mantenimiento esta área anatómica: debridamiento mecánico noquirúrgico y quirúrgico, plastia furcal, procedimientos de tunelización, hemisecciones, amputaciones radiculares y tratamiento regenerativos. Hamp, Nyman y Lindhe (5) en 1975 describieron la plastía furcal como uno de los tratamientos para tratar lesio- 
nes de Clase I en la clasificación propuesta por ellos. El tratamiento de plastia furcal según Hamp consiste en el desbridamiento (eliminación de tejido de granulación y depósitos bacterianos dentro de la furcación), odontoplastía (remoción de sustancia dentaria para eliminar o reducir la extensión de las pared horizontal del defecto de la furcación) y osteoplastia (recontorneado del defecto óseo adyacente)

El propósito del presente reporte de caso es mostrar la eficacia de la plastia furcal como tratamiento para lesiones de furcación Clase I.

\section{Reporte de caso}

Paciente de sexo femenino, mestiza de 69 años fue a consulta por presentar movilidad dentaria en el sector anteroinferior y sangrado de sus encías al momento del cepillado. Los antecedentes sistémicos personales obtenidos por medio de la anamnesis no fueron contribuyentes (Fig.1).

Al examen clínico presentaba profundidades de sondaje $>4 \mathrm{~mm}$ localizadas principalmente en molares, sangrado al sondaje, movilidad dentaria, falta de encía queratinizada con inserción de frenillo inferior izquierdo aberrante a nivel de pza 34, índice de higiene oral según O'Leary de $75 \%$ y de sangrado según Ainamo de $45 \%$. Así mismo, presentaba lesiones de furcación en molares superiores e inferiores Clase I (3 mm) según la clasificación de Hamp (5). Al examen radiológico se observó pérdida de hueso alveolar localizada, de moderada a severa. Se diagnosticó el caso como periodontitis crónica moderada localizada (Fig. 2 y 3).

Se realizó la fase inicial de la terapia periodontal que consistió en instrucción de higiene oral y raspado y alisado radicular. Así mismo se procedió a ferulizar las piezas anteroinferiores. A la reevaluación de la fase inicial se observó la presencia de profundidades de sondaje mayor a $4 \mathrm{~mm}$. en molares superiores, inferiores y a nivel de pieza 34, así como también la persistencia de signos clínicos de inflamación a nivel de encía marginal y papilar.

Debido a la pobre respuesta frente al tratamiento se decidió realizar una fase complementaria consistente en el raspado y alisado radicular de las piezas con bolsas periodontales persistentes.

Se utilizó para el debridamiento de las superficies radiculares afectadas curetas específicas (Gracey, Mc Call, Gracey-minifive) e instrumentación con ultrasonido en 4 sesiones de 1 hora cada una en el lapso de 10 días.

Se realizó el control de placa bacteriana semanalmente, sin embargo, a la reevaluación a los 21 días, se observó nuevamente la persistencia de profundidades al sondaje patológicas, placa y sangrado.

Se procedió a programar una fase quirúrgica en la cual se realizó la plastía furcal con colgajo reposicionado a nivel de las piezas 17 y 16, injerto gingival libre para aumento de grosor de encía queratinizada y colgajo de acceso a nivel de pieza 47.

Los procedimientos realizados en la cirugía de plastía furcal con colgajo reposicionado fueron : asepsia y antisepsia, colocación de campos estériles, anestesia infiltrativa a nivel de fondo de surco y mucosa palatina de pzas 17-15, Incisión en almohadilla retromolar, Incisión intrasurcal vestibular y palatino de pza 17-15, decolado a espesor total, eliminación de tejido de granulación, instrumentación ultrasónica de pza 17 y 16 más raspado y alisado radicular, osteoplastía y odontoplastía con fresa de pulido grano extrafino, relleno de furcación tipo I con hueso autólogo obtenido de la tuberosidad del maxilar, reposicionamiento del colgajo. Lavado con $\mathrm{NaCl}$ al $0,9 \%$. Sutura simple (Fig. 4).

A la reevaluación de la fase quirúrgica se pudo observar la mejoría de las zonas operadas, así como la mejora del control de placa y reducción de los signos clínicos de infla-

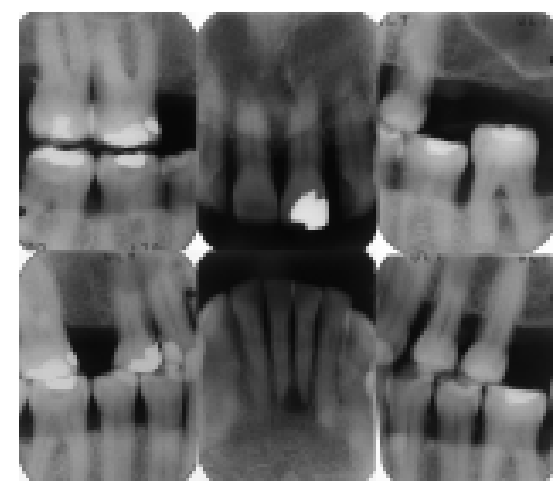

Fig. 2. Set radiográfico. Se observa pérdida ósea alveolar moderada a nivel interproximal.

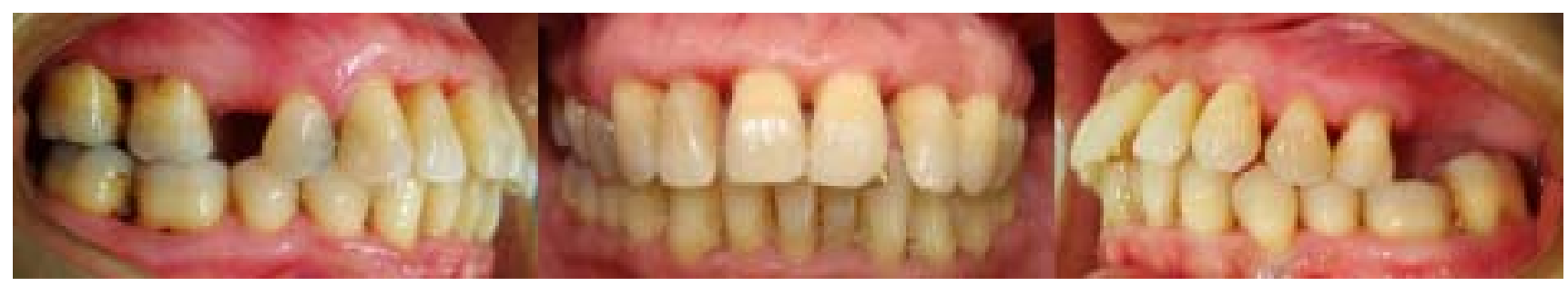

Fig. 1. Fotografias iniciales. A la evaluación se observa recesión de tejidos marginales y pérdida de papilas dentales. 


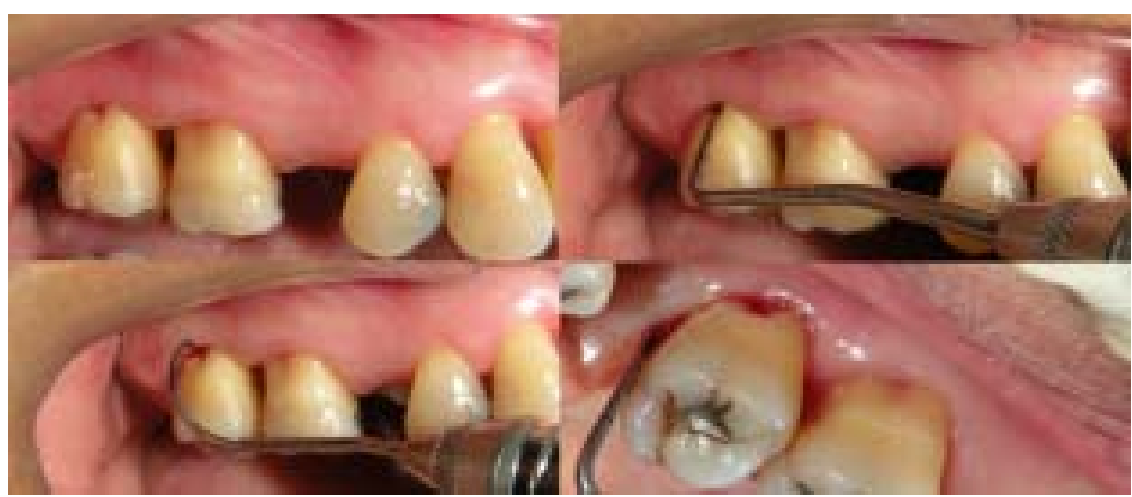

Fig. 3. Fotografias clínicas de piezas 17 y 16 . A la evaluación se observa sangrado y profundidad al sondaje en sentido horizontal mayor a $4 \mathrm{~mm}$.

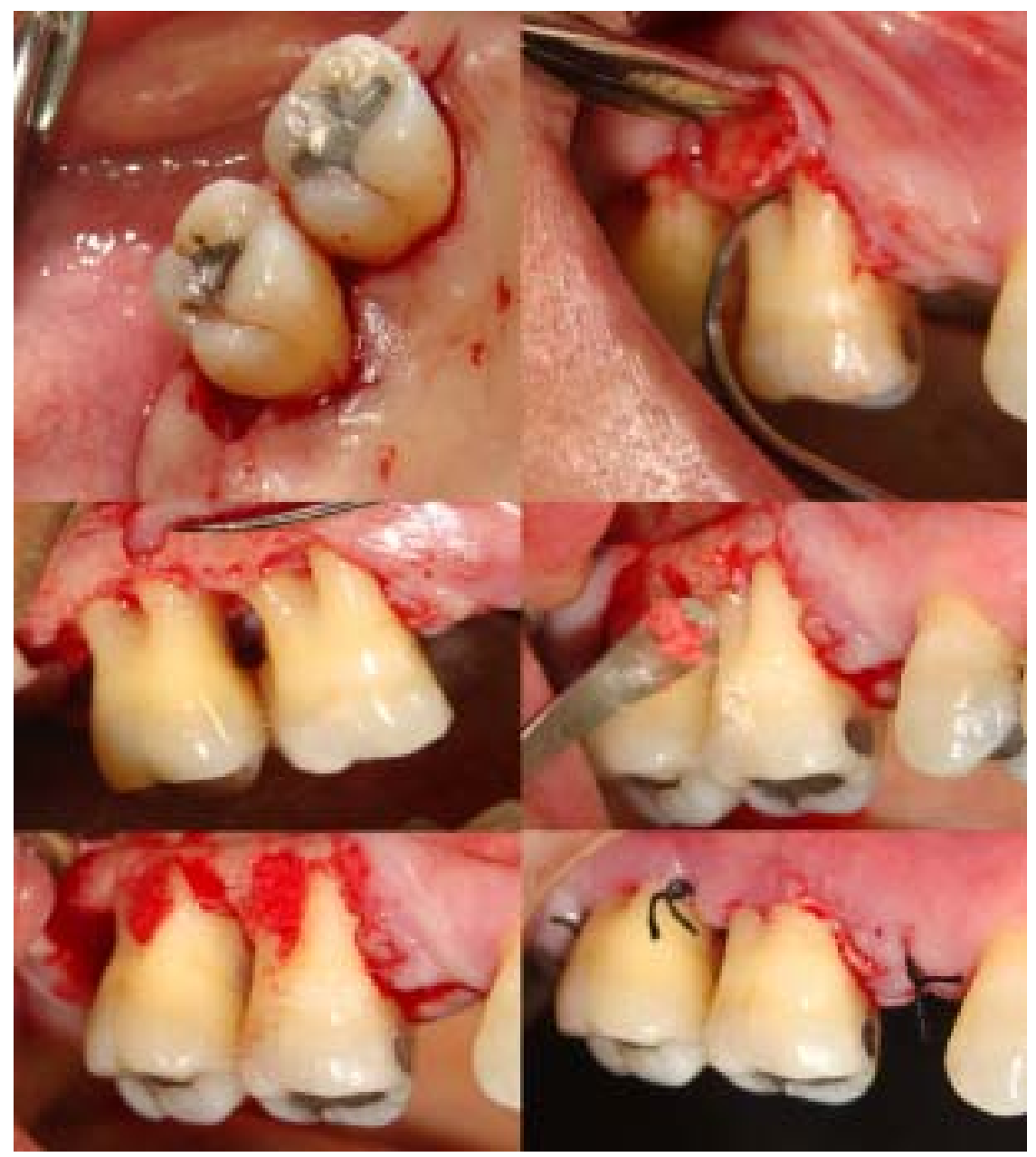

Fig. 4. Fotografias clínicas intraoperatorias. Se puede observar el compromiso de furcación. Se procedió a la odontopastía en sentido horizontal de la zona, así como el relleno óseo con hueso autólogo procedente de zona adyacente.

mación (Fig. 5).

\section{Discusión}

Los resultados obtenidos en el presente caso clínico demuestran que en casos de periodontitis crónica moderada en piezas dentarias con
1989, y Claffey y Egelberg (8) en 1994.

Las dimensiones reducidas de las entradas de las furcaciones pueden comprometer el éxito del tratamiento periodontal, por consecuente es necesario una buena accesibilidad e instrumentos que se adecuen a la morfología de esta área anatómica. Chiu et al. (12) en 1991 encontraron que el $79 \%$ de las entradas de furcación vestibular en molares superiores son menores a $0,75 \mathrm{~mm}$, siendo $0,76 \mathrm{~mm}$ el ancho de una cureta Gracey nueva. Resultados similares fueron reportados por Dos Santos et al. (13) en 2009 donde el $36 \%$ de la entrada de furcación de las molares superiores era menor a $0,60 \mathrm{~mm}$, encontrando que los anchos promedios de las curetas Gracey 5/6, Mc Call 17/18 y Gracey-minifive 5/6 eran de 0,67 $\pm 0,06 \mathrm{~mm} ; 0,63 \pm 0,12 \mathrm{~mm}$; $0,63 \pm 0,05 \mathrm{~mm}$ respectivamente.

Parashis et al. (14) evaluaron la efectividad del raspado y alisado radicular (RAR) en áreas de furcación. Su investigación evaluó tres grupos de estudio: RAR sin colgajo, RAR con colgajo y RAR con colgajo más pulido con fresa de grano extrafino. Los resultados obtenidos demostraron que sólo el 12,5\% de las superficies de furcación tratadas con RAR y sin colgajo de acceso se encontraban totalmente libres de placa, mientras el 25\% de las superficies tratadas con RAR y colgajo de acceso estaban totalmente libres de placa. Los resultados mejoraban hasta un 38\% cuando una fresa de pulido era utilizada en esta área anatómica.

\section{Conclusiones}

- La evidencia disponible indica que la terapia no-quirúrgica debe ser complementada por terapia resectiva y/o regenerativa en pie- 


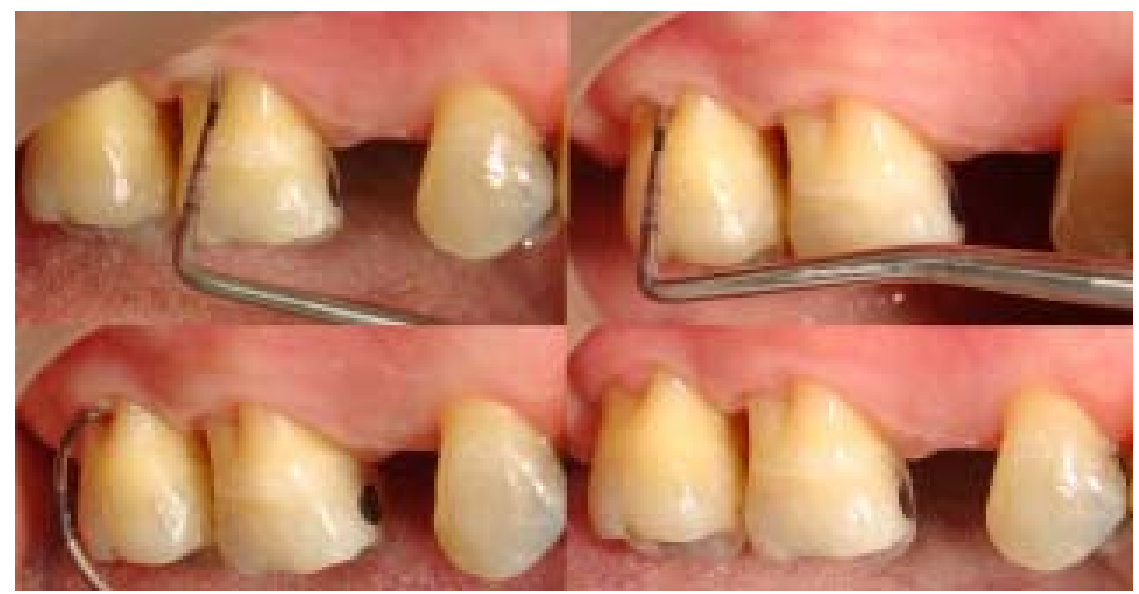

Fig. 5. Fotografias clínicas de control a los 6 meses. Se observa la reducción de la profundidad al sondaje y ausencia de signos clínicos de inflamación.

zas dentarias con compromiso de furcación.

- El objetivo de la plastia furcal es disminuir la dimensión horizontal de la furcación y estructuras adyacentes con el objetivo de mejorar la arquitectura de las mismas y facilitar el acceso a la higiene.

- La plastia furcal se muestra como una opción efectiva para la mejora de las condiciones periodontales en dientes con lesión de furcación Clase I.

\section{Referencias bibliográficas}

1. Socransky SS, Haffajee AD. Periodontal microbial ecology. Periodontol 2000. 2005; 38:13587.

2. Axelsson P, Nyström B, Lindhe $\mathrm{J}$. The long-term effect of a plaque control program on tooth mortality, caries and periodontal disease in adults. Results after 30 years of maintenance. J Clin Periodontol. 2004; 31(9):749-57.

3. Fleischer HC, Mellonig JT, Brayer WK, Gray JL, Barnett
JD. Scaling and root planing efficacy in multirooted teeth. J Periodontol. 1989; 60(7):402-9.

4. Lang NP, Cumming BR, Löe H. Toothbrushing frequency as it relates to plaque development and gingival health. J Periodontol. 1973; 44(7):396-405.

5. Hamp SE, Nyman S, Lindhe J. Periodontal treatment of multirooted teeth. Results after 5 years. J Clin Periodontol. 1975; 2(3):126-35.

6. Nordland P, Garrett S, Kiger R, Vanooteghem R, Hutchens LH, Egelberg J. The effect of plaque control and root debridement in molar teeth. J Clin Periodontol. 1987; 14(4):231-6.

7. Loos B, Nylund K, Claffey N, Egelberg J. Clinical effects of root debridement in molar and non-molar teeth. A 2-year followup. J Clin Periodontol. 1989; 16(8):498-504.

8. Claffey N, Egelberg J. Clinical characteristics of periodontal sites with probing attachment loss following initial periodontal treatment. J Clin Periodontol. 1994; 21(10):670-9.

9. Hirschfeld L, Wasserman B. A long-term survey of tooth loss in 600 treated periodontal patients. J Periodontol. 1978; 49(5):22537.

10. McFall WT Jr. Tooth loss in 100 treated patients with periodontal disease. A long-term study. J Periodontol. 1982; 53(9):539-49.

11. Goldman MJ, Ross IF, Goteiner D. Effect of periodontal therapy on patients maintained for 15 years or longer. A retrospective study. J Periodontol. 1986; 57(6):347-53.

12.Chiu BM, Zee KY, Corbet EF, Holmgren CJ. Periodontal implications of furcation entrance dimensions in Chinese first permanent molars. J Periodontol. 1991; 62(5):308-11.

13.dos Santos KM, Pinto SC, Pochapski MT, Wambier DS, Pilatti GL, Santos FA. Molar furcation entrance and its relation to the width of curette blades used in periodontal mechanical therapy. Int J Dent Hyg. 2009; 7(4):263-9.

14.Parashis AO, AnagnouVareltzides A, Demetriou N. Calculus removal from multirooted teeth with and without surgical access. II. Comparison between external and furcation surfaces and effect of furcation entrance width. J Clin Periodontol. 1993; 20(4):294-8. 